\title{
An Encoder-Decoder Framework Translating Natural Language to Database Queries
}

\author{
Ruichu Cai ${ }^{1}$, Boyan Xu ${ }^{1}$, Zhenjie Zhang ${ }^{2}$, Xiaoyan Yang ${ }^{2}$, Zijian Li $^{1}$, Zhihao Liang ${ }^{1}$ \\ ${ }^{1}$ Faculty of Computer, Guangdong University of Technology, China \\ ${ }^{2}$ Singapore R\&D, Yitu Technology Ltd. \\ cairuichu@gmail.com, hpakyim@gmail.com, zhenjie.zhang@yitu-inc.com \\ xiaoyan.yang@yitu-inc.com, leizigin@gmail.com, zhihaolzh95@gmail.com
}

\begin{abstract}
Machine translation is going through a radical revolution, driven by the explosive development of deep learning techniques using Convolutional Neural Network $(\mathrm{CNN})$ and Recurrent Neural Network (RNN). In this paper, we consider a special case in machine translation problems, targeting to convert natural language into Structured Query Language (SQL) for data retrieval over relational database. Although generic CNN and RNN learn the grammar structure of SQL when trained with sufficient samples, the accuracy and training efficiency of the model could be dramatically improved, when the translation model is deeply integrated with the grammar rules of SQL. We present a new encoder-decoder framework, with a suite of new approaches, including new semantic features fed into the encoder, grammar-aware states injected into the memory of decoder, as well as recursive state management for sub-queries. These techniques help the neural network better focus on understanding semantics of operations in natural language and save the efforts on SQL grammar learning. The empirical evaluation on real world database and queries show that our approach outperform state-of-the-art solution by a significant margin.
\end{abstract}

\section{Introduction}

Machine translation is known as one of the fundamental problems in machine learning, attracting extensive research efforts in the last few decades [Koncar and Guthrie, 1997; Castano et al., 1997]. In recent years, with the explosive development of deep learning techniques, the performance of machine translation is dramatically improved, by adopting convolutional neural network [Gehring et al., 2017] or recurrent neural network [Cho et al., 2014; Wu et al., 2016; Zhou et al., 2016]. The growing demands of computerhuman interaction in the big data era, however, is now looking for additional support from machine translation to convert human commands into actionable items understand- able to database systems [Giordani and Moschitti, 2012; Li and Jagadish, 2014; Mou et al., 2017; Popescu et al., 2003; Rabinovich et al., 2017; Yin and Neubig, 2017], in order to ease the efforts of human users on learning and writing complicated Structured Query Language (SQL). Our problem is known to be more challenging than the traditional semantic parsing problem, e.g., latest SCONE dataset involving context-dependent parsing [Long et al., 2016], because of the high complexity of database querying language. Given a complex real world database, e.g., Microsoft academic database [Roy et al., 2013], it contains dozens of tables and even more primary-foreign key column pairs. A short natural language question, such as "Find all IJCAI 2018 author names" must be converted into a SQL query with more than 10 lines, because the result query involves four tables.

Recently, a number of research works attempt to apply neural network approaches on data querying, such as [Neelakantan et al., 2016; Yin et al., 2016], which target to generate data processing results by directly linking records in data tables to the semantic meanings of the natural language questions. There are two major limitations rooted at the design of their solutions. First, such methods are not scalable to big data tables, since the computation complexity is almost linear to the cardinality of the target data tables. Second, the conversion results of such methods are not reusable when a database is updated. The original natural language queries must be recalculated from scratch, in order to generate results on a new table or newly incoming records. The key to a more scalable and extensible solution is to transform original natural language queries into SQL queries instead of query answers, such that the result SQL queries are simply reusable on all tables of arbitrary size at any time.

Technically, we opt to employ encoder-decoder framework as the underlying translation model, based on Recurrent Neural Network and Long Short Term Memory (LSTM) [Hochreiter and Schmidhuber, 1997]. Basically, in the encoder phase, the neural network recognizes and maintains the semantic information of the natural language question. In the decoder phase, it outputs a new sequence in another language based on the information maintained in the hidden states of the neural network. Encoder-decoder framework has outperformed conventional approaches over generic translation tasks for vari- 
ous pairs of natural languages. When the output domain is a structured language, such as SQL, although encoder-decoder framework is supposed to learn the grammar structure of SQL when given sufficient training samples, the cost is generally too high to afford. It spends most of the computation power on grammar understanding, but only little effort on the semantical interpretation of original questions. Even given sufficient training data, the output of standard encoder-decoder may not fully comply with SQL standard, potentially ruining the utility of the result SQL queries on real databases.

In this paper, we propose a new approach smoothly combining deep learning techniques and traditional query parsing techniques. Different from recent studies with similar strategy [Iyer et al., 2017; Rabinovich et al., 2017; Yin and Neubig, 2017], we include a suite of new methods specially designed for structured language outputting. On the encoder phase, instead of directly feeding word representations into the neural network, we inject a few new bits into the memory of the neural network based on languageaware semantical labels over the input words, such as table names and column names in SQL. These additional dimensions are not directly learnable by language models, but explicitly recognizable based on the properties of the structured language. On the decoder phase, we insert additional hidden states in the memory layer, called grammatical states, which indicate the states of the translation output in terms of Backus-Naur Form (BNF) of SQL. To handle the complexity behind schema-relevant information, our system generates two types of dependency and masking mechanisms to better capture the constraints based on SQL grammar as well as database schema. We also allow the neural network to recursively track the grammatical state when diving into subqueries, such that necessary information is properly maintained even when nested queries are finished.

The core contributions of the paper are summarized as follows: 1) we present an enhanced encoder-decoder framework deeply integrated with known grammar structure of SQL; 2) we discuss the new techniques included in encoder and decoder phases respectively on grammar-aware neural network processing; 3) we evaluate the usefulness of our new framework on synthetic workload of real world database for natural language querying.

\section{Related Work}

The emergence of deep learning techniques, particularly recurrent neural network for sequential domain, enables the machine learning models to build such complicated dependencies, and greatly enhance the translation accuracy. Encoderdecoder framework is known as a typical RNN framework designed for machine translation [Cho et al., 2014; Sutskever et al., 2014]. On the other hand, convolutional neural network models are recently recognized as an effective alternative to recurrent neural network model for machine translation tasks. In [Gehring et al., 2017], Gehring et al. show that convolution allows the machine learning system to better train translation model by using GPUs and other parallel computation techniques.

In last two years, researchers are turning to adopt recur- rent neural network for automatic data querying and programming based on natural language inputs, which aims to translate original natural language into programs and data querying results. Semantic parsing, for example, is the problem of converting natural language into formal and executable logics. In last two years, sequence-to-sequence model is becoming state-of-the-art solution of semantic parsing [Xiao et al., 2016; Dong and Lapata, 2016; Guu et al., 2017]. While most of the existing studies exploit the availability of human intelligence for additional labels [Jia and Liang, 2016; Liang, 2016], our approach learns the translation with inputoutput sample pairs only. While masking is proposed in the literature for symbolic parsing by storing key-variable pairs in the memory [Liang et al., 2017], the masking technique proposed in this paper supports more complex operations, covering both short-term and long-term dependencies. Moreover, we hereby emphasize that the grammar structure of SQL is known to be much more complicated than the logical forms used in semantic parsing.

Besides of semantic parsing, researchers are also attempting to generate executable logics by directly linking the semantic interpretation of the input natural language and the records in the database. Neural networks are employed to identify appropriate operators [Neelakantan et al., 2016], while distributed representations are used [Mou et al., 2017; Yin et al., 2016] to columns, rows and records in the data table. As pointed out in the introduction, such approaches do not scale up in terms of the data size, and the outputs are not reusable over a new data table or updated table with new records.

Recently, [Iyer et al., 2017; Rabinovich et al., 2017; Yin and Neubig, 2017] try to integrate grammar structure into sequence-to-sequence model for data processing query generation. These studies share common idea of our paper on tracking grammar states of the output sequence. Our approach, however, differentiates on two major points. Firstly, we design consistent and systematic approach based on grammar rule (i.e., centered at non-terminal symbols in BNF) for both encoder and decoder phases. Secondly, we include both short-term and long-term dependency in output word screening based on grammar state, exploiting the information from the schemas of the databases. These features bring significant robustness improvement.

\section{Overview}

In this paper, we formulate the translation process as a mapping from a natural language domain $\mathbb{N}$ to a structured language domain $\mathbb{S}$, i.e., $\mathbb{N} \mapsto \mathbb{S}$. The input from $\mathbb{N}$ is a natural language sentence, $N=\left(w_{1}, w_{2}, \ldots, w_{L_{N}}\right)$ with every word $w_{i}$ from a known dictionary $D_{\mathbb{N}}$. Similarly, the output of the mapping is another text sequence, $S=\left(w_{1}, w_{2}, \ldots, w_{L_{S}}\right)$, in a structured language domain, e.g., SQL on a relational database, with dictionary $D_{\mathbb{S}}$. The goal of the translation learning is to reconstruct the mapping, based on given samples of the translation, i.e., a training set with natural language and corresponding queries $T=\left\{\left(N_{1}, S_{1}\right), \ldots,\left(N_{n}, S_{n}\right)\right\} \subset$ $\mathbb{N} \times \mathbb{S}$.

Encoder-decoder framework [Sutskever et al., 2014] is the 


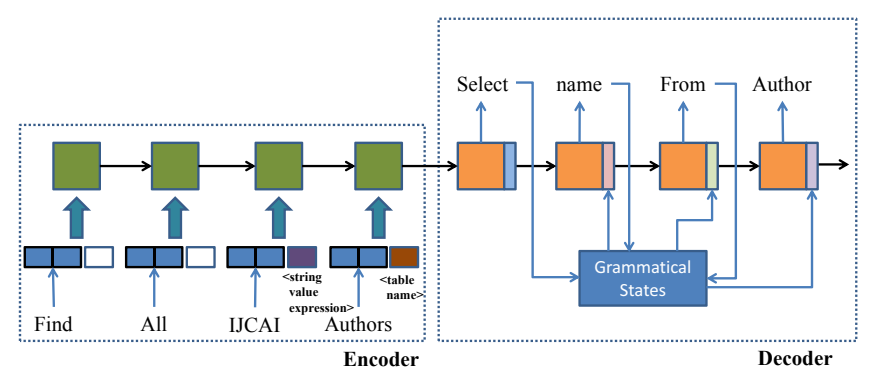

Figure 1: A running example of our new Encoder-Decoder framework. The encoder phase accepts new semantic labels of the input words based on text analysis. The decoder phase employs additional augmented memory controlled by grammatical state.

state-of-the-art solution to general machine translation problem between arbitrary language pairs. As is shown in Figure 1 , there are two phases in the transformation from an input sequence to output sequence, namely encoder phase and $d e$ coder phase. The encoder phase mainly processes the input sequence, extracts key information of the input sequence and appropriately maintains them in the hidden layer, or memory in another word, of the neural network. The decoder phase is responsible for output generation, which sequentially selects output words in its dictionary and updates the memory state accordingly.

In this paper, we propose a variant encoder-decoder model, with new features designed based on the purpose of converting natural language into executable and structured language. The general motivations of these new features are also presented in Figure 1. In the encoder phase, besides of the vectorized representations of the input words, we add a number of additional binary bits into the input vector to the neural network. These binary bits are used to indicate the possible semantical meaning of these words. In our example, the word "IJCAI" is labeled as string value expression and the word "Authors" is marked as a potential column name in the table. Note that such information is not directly inferrable by a distributed representation system, e.g., [Mikolov et al., 2013]. In the decoder phase, we also add new binary bits to the hidden memory layer. These states are not manipulated by the neural network, but by certain external control logics. Given the history of the output words, the external logics calculate the grammatical status of the output sequence. These augmented grammatical status is further utilized to mask candidate words for outputting. as well as feedforward features to the hidden layer of neural network. This mechanism enables our system output executable SQL at any time and enhances the learnability of the neural network.

Different from recent studies [Rabinovich et al., 2017; Yin and Neubig, 2017], we utilize Backus Normal Form (BNF) to generate grammatical state tracking. A BNF specification of a language is a set of derivation rules, consisting of a group of symbols and expressions. There are two types of symbols, terminal symbols and non-terminal symbols. If a symbols is non-terminal, corresponding expression contains one or more sequences of symbols. These sequences are separated by the vertical bars, each of which

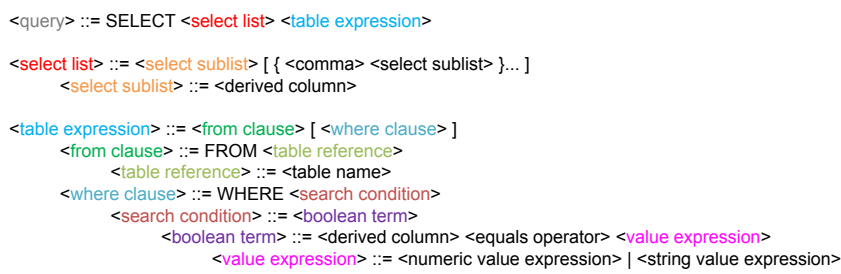

Figure 2: BNF of grammar structure of selection queries in SQL-92 standard. In the derivation rules, we use \langle\rangle to indicate a symbol, [] to indicate an option and \{\} to indicate a block of symbols.

is a possible substitution for the symbol on the left. Terminal symbols never appear on the left side of any expression. In Figure 2, we present the BNF of SQL-92, with 〈query as the root symbol. All colored symbols, e.g., 〈table expression $\rangle$, are non-terminal symbols, and symbols in black, e.g., $\langle$ numeric value expression $\rangle$, are terminal symbols. Theoretically, the language is context-free, if it could be written in form of BNF, and therefore deterministically verified by a push-down automaton. Given the BNF of SQL in Figure 2, parsers in relational database systems can easily track the grammatical correctness of an input SQL query by scanning the query from beginning to end. Although grammar tracking strategy is similar to [Rabinovich et al., 2017; Yin and Neubig, 2017], we employ short-term and long-term dependencies to accurately mask words based on both SQL grammar and database schema.

\section{Techniques}

Encoder Processing: The key of encoder phase in the framework is to digest the original natural language input and put the most important information in the memory before proceeding to the decoder phase. In order to extract useful information from the words in the sentence, we propose to extract additional semantic features that link the original words to the semantics of the grammatical structure of the target language.

We generate a group of labels based on the BNF of the target language $\mathbb{S}$. Specifically, each label corresponds to a terminal symbol in the BNF. Based on the BNF in Figure 2, there are four terminal symbols with corresponding labels.

$\langle$ Derived column $\rangle$ : refers to words used to describe the columns specified in the database query, e.g., the word "name" in Figure 1.

$\langle$ Table reference $\rangle$ : refers to words used to describe the tables specified in the database query, e.g., the word "author".

$\langle$ Value expression $\rangle$ : refers to words containing numeric values used to describe the conditions in the database query.

$\langle$ String expression $\rangle$ : refers to words containing string values used to describe the conditions in the database query, e.g., the word "IJCAI" in Figure 1.

Given a small group of samples, we manually label the words with these four label types and employ conditional random fields (CRFs) [Lafferty et al., 2001] to build effective classifiers for these labels.

Decoder Processing: We employ two different techniques in the decoder phase, including the embedding of grammar state in the hidden layer and the masking of word outputs. 


\begin{tabular}{|c|c|c|c|c|}
\hline Table & \multicolumn{3}{|l|}{ Attributes/Columns } & \\
\hline author & \multicolumn{3}{|c|}{ aid, name, photo, age, homepage, oid } & \\
\hline publication & \multicolumn{4}{|c|}{ pid, title, abstract, year, cid, jid, reference_num, citation_num, doi } \\
\hline \multicolumn{2}{|l|}{ step 1} & \multicolumn{3}{|c|}{ Short-term Dependency } \\
\hline \multirow{2}{*}{\multicolumn{2}{|c|}{ Output: Select }} & Current State & Current Word & Next Word Mask \\
\hline & & <query & SELECT & 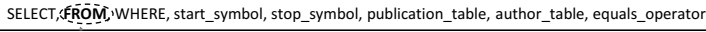 \\
\hline & & \multicolumn{3}{|c|}{ Long-term Dependency } \\
\hline \multirow{2}{*}{ <query> } & & Active Symbol & Current Word & Next Wordi Mask \\
\hline & & & & $\ldots \ldots+\ldots *^{* *^{*}}$ \\
\hline Step 2 & \multicolumn{4}{|c|}{ 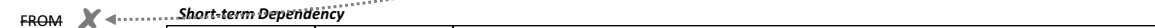 } \\
\hline \multirow{2}{*}{\multicolumn{2}{|c|}{ Output: Select name $\vee$}} & Current State & Current Word & Next Word Mask \\
\hline & & <derived column> & name & $\begin{array}{l}\text { SELECT, WHERE, start_symbol, stop_symbol, publication_table, author_table, equals_operator, } \\
\text { publication_column, name }\end{array}$ \\
\hline \multirow{3}{*}{ <query> } & \multirow{3}{*}{$\begin{array}{l}<\text { derived } \\
\text { column }\end{array}$} & \multicolumn{3}{|c|}{ Long-term Dependency } \\
\hline & & Active Symbol & Current Word & Next Word Mask \\
\hline & & <derived column> & name & publication_table, publication_column, name \\
\hline \multicolumn{2}{|c|}{$\begin{array}{l}\text { Step } 3 \\
\quad \text { Output: Select name from }\end{array}$} & \multicolumn{3}{|c|}{ Short-term Dependency } \\
\hline \multirow{2}{*}{\multicolumn{2}{|c|}{ Output: Select name from }} & Current State & Current Word & Next Word Mask \\
\hline & & <from clause> & FROM & $\begin{array}{l}\text { SELECT, WHERE, start_symbol, stop_symbol, equals_operator, publication_column, author_column, } \\
\text { FROM }\end{array}$ \\
\hline \multirow{3}{*}{$\begin{array}{l}\text { <derived } \\
\text { column> }\end{array}$} & \multirow{3}{*}{$\begin{array}{l}\text { sfrom } \\
\text { clause> }\end{array}$} & \multicolumn{3}{|c|}{ Long-term Dependency } \\
\hline & & Active Symbol & Current Word & Next Word Mask \\
\hline & & <derived column> & name & publication_table, publication_column, name \\
\hline \multirow{3}{*}{\multicolumn{2}{|c|}{$\begin{array}{l}\text { Step } 4 \\
\quad \text { Output: Select name from author }\end{array}$}} & \multicolumn{3}{|c|}{ Short-term Dependency } \\
\hline & & Current State & Current Word & Next Word Mask \\
\hline & & <table reference > & author & $\begin{array}{l}\text { SELECT, FROM, start_symbol, publication_table, author_table, equals_operator, author_column } \\
\text { publication_column }\end{array}$ \\
\hline \multirow{4}{*}{$\begin{array}{l}\text { <from } \\
\text { clause> }\end{array}$} & \multirow{4}{*}{$\begin{array}{l}<\text { table } \\
\text { reference }\end{array}$} & \multicolumn{3}{|c|}{ Long-term Dependency } \\
\hline & & Active Symbol & Current Word & Next Word Mask \\
\hline & & <derived column> & name & publication_table, publication_column, name \\
\hline & & <table reference > & author & publication_table, publication_column \\
\hline
\end{tabular}

Figure 3: A running example of the decoder phase assuming simple selection query over two tables "author" and "publication": in the first step, given the grammar state "Query" and current word "SELECT", the decoder adds word masking by finding S1, a rule of short-term dependency. The word mask blocks the output of word "FROM" in next step. After outputting the word "name", the decoder further adds new word masking by identifying L1 in long-term dependency rules. Because of the word masking from L1, the decoder is only allowed to output "author" as the table for querying.

Basically, given a particular word in the output sequence, the grammar state of the word is the last expression of BNF this word fits in. When a parser interprets a SQL query, it selects the candidate expression for the words based on the structure of BNF. In the example shown in Figure 3, the parser enters state derived column when it encounters word "name" in step 2. To facilitate grammar state tracking, we use a binary vector structure to represent all possible states. The length of the vector is identical to the number of expressions in the $\mathrm{BNF}$ of $\mathbb{S}$. Each binary bit in the vector denotes if a particular expression is active based on the parser. When reading a new word of the output of the decoder, the SQL parser updates the grammar vector to reflect the semantic meaning of the word. The grammar state is used not only for state tracking but also for the update of the memory of the neural network. Let $g_{t}$ denote the grammar status information at time $t$. To incorporate $g_{t}$ into the model, the memory of the neural network is updated as follows:

$$
\begin{aligned}
f_{t} & =\sigma_{g}\left(W_{f} x_{t}+U_{f} h_{t-1}+V_{f} g_{t-1}+b_{f}\right) \\
i_{t} & =\sigma_{g}\left(W_{i} x_{t}+U_{i} h_{t-1}+V_{i} g_{t-1}+b_{i}\right) \\
o_{t} & =\sigma_{g}\left(W_{o} x_{t}+U_{o} h_{t-1}+V_{o} g_{t-1}+b_{o}\right) \\
c_{t} & =f_{t} \otimes c_{t-1}+i_{t} \otimes \sigma_{c}\left(W_{f} x_{t}+U_{f} h_{t-1}+V_{f} g_{t-1}+b_{f}\right) \\
h_{t} & =o_{t} \otimes \sigma\left(c_{t}\right)
\end{aligned}
$$

where $\otimes$ indicates element-wise multiplication operation.

\begin{tabular}{|l||l|l||l|}
\hline ID & State & Current Word/Symbol & Next Word/Symbol \\
\hline \hline S1 & $\langle$ query $\rangle$ & SELECT & $\langle$ derived column $\rangle$ \\
\hline S2 & $\langle$ select list $\rangle$ & $\langle$ derived column $\rangle$ & $\langle$ comma, FROM \\
\hline S3 & $\langle$ select list $\rangle$ & $\langle$ comma $\rangle$ & $\langle$ derived column $\rangle$ \\
\hline S4 & $\langle$ from clause $\rangle$ & FROM & $\langle$ table name $\rangle$ \\
\hline S5 & $\langle$ from clause $\rangle$ & $\langle$ table name $\rangle$ & WHERE, Stop_symbol \\
\hline S6 & $\langle$ where clause $\rangle$ & WHERE & $\langle$ derived column $\rangle$ \\
\hline S7 & $\langle$ where clause $\rangle$ & $\langle$ derived column $\rangle$ & $\langle$ equals operator $\rangle$ \\
\hline S8 & $\langle$ where clause $\rangle$ & $\langle$ equals operator $\rangle$ & $\langle$ value expression $\rangle$ \\
\hline S9 & $\langle$ where clause $\rangle$ & $\langle$ value expression $\rangle$ & Stop_symbol \\
\hline
\end{tabular}

Table 1: Partial rules of Short-term Dependencies.

\begin{tabular}{|l||l|l||l|}
\hline ID & Symbol & Current Word & Long Term Word Mask \\
\hline L1 & $\langle$ derived column $\rangle$ & name & publication_table/column, name \\
\hline L2 & $\langle$ table name $\rangle$ & author & publication_table/column \\
\hline
\end{tabular}

Table 2: Partial rules of Long-term Dependencies.

Output Words Masking: In the decoder, there are two types of word masks used to filter out invalid words for outputing, which are mainly based on short-term dependencies and long-term dependencies respectively. At each step, the decoder chooses one rule from candidate short-term dependencies, e.g., rules in Table 1 , and possibly multiple rules from candidate long-term dependencies, e.g., rules in Table 2. The short-term dependency rule is updated according to the current grammar state as well as the last output word from the 
decoder. In Table 1, the columns of "State" and "Current Word/Symbol" are used for rule matching, while the column "Next Word/Symbol" indicates all valid output words in next step of the decoder. Once the decoder identifies a matching rule, it generates a mask on the dictionary to block the output of words not allowed by the rule. Long-term dependencies are updated based on the active symbols chosen by the SQL parser, maintained in the grammar state vector. For each active symbol, the decoder includes a rule from all long-term dependency rules, e.g., Table 2, by matching on "Symbol" and "Word". Given the rule, the decoder generates the output word mask accordingly. The rules for long-term dependencies are removed from the decoder, only when the corresponding symbol turns inactive.

We use a binary vector $s$ to indicate the masks generated by the single rule of short-term dependency, and $l_{i}$ for the $i$-th mask generated by the rule of long-term dependencies. Given these masks, the word selection process in the decoder is modified as:

$$
y_{t}=\sigma_{y}\left(W_{y} h_{t}+b_{y}\right) \otimes s \otimes l_{1} \ldots \otimes l_{L},
$$

where $L$ is the number of active long-term dependency rules.

In Figure 3, we present a detailed running example on the evolution of the active rules and corresponding masks, to elaborate the effect of combining the neural network and the grammar state transition. Following the example in Figure 1 , the query attempts to retrieve names from the author table, with the grammar states and masks updated based on the descriptions above.

Dependency Rule Generation: The automatic generation of rules for short-term dependencies and long-term dependencies are different. Due to the limited space, we only provide a sketch of the generation methods in the current version.

For short-term dependency, the framework identifies the reachable terminal symbols for every pair of symbol and word. Consider S1 in Table 1. Given the symbol 〈query〉 and word output "SELECT", the only matching expression in $\mathrm{BNF}$ is $\langle$ query $\rangle:=$ SELECT $\langle$ select list $\rangle\langle$ table expression $\rangle$. The following symbol is $\langle$ select list $\rangle$. Since $\langle$ select list $\rangle$ is not a terminal symbol, we iterate over the BNF to find the terminal symbols to generate in next step. In this case, we reach the terminal symbol 〈derived column〉 and thus insert it into the fourth column of S1 in Table 1.

For long-term dependency, the framework must combine the BNF as well as the schema of the database. Currently, we only consider 〈derived column $\rangle$ and $\langle$ table name $\rangle$, which forbid the adoption of non-relevant tables and columns in the rest of the SQL query.

\section{Experiments}

Workload Preparation: We run our experiments on three databases, namely Geo880, Academic and IMDB. The workload on Geo 880 is generated by converging logical form outcomes to equivalent relational table and SQL queries. Academic database has 17 tables, collected by Microsoft Academic Search [Roy et al., 2013]. This database is employed in the experiments of [Li and Jagadish, 2014]. IMDB has 3 tables, containing records of 3,654 movies, 4,370 actors and 1,659 directors. On Academic and $I M D B$, we generate SQL query workloads and ask volunteers to label the queries with natural language descriptions. Specifically, two types of workloads are generated, namely Select workload and Join workload. The queries in Select and Join (with 4 concrete aggregation operators for AGG, including Min, Max, Average and Count) are in the following two forms respectively:

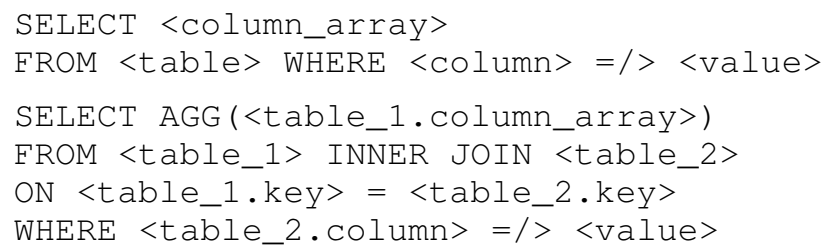

Given the standard forms of the queries above, we generate concrete queries by randomly selecting the tables and columns without replacement. For each combination of tables and columns, we randomly select values for the conditions in the queries. By manually filtering out meaningless queries, we generate 35 queries on Academic database and 75 queries on $I M D B$ database. Each query is manually labeled by at least 5 independent volunteers. Given a pair of natural language description label and query, we further generalize it to a group of variant queries, by modifying the search conditions in where clauses. Consequently, we get 1,456 (376 select query and 1,080 join query) pairs of samples on Academic database and 2,103 (1,082 select query and 1,021 join query) pairs of samples on $I M D B$ database. We also build a Mixed workload, by simply combining all samples from both Select and Join workloads. We reuse the queries and natural language descriptions in Geo880 database, and use the standard training/test split as in [Iyer et al., 2017].

Baseline Approaches: We employ two state-of-the-art and representative approaches as baseline in our experiments, including NLP translation model NMT [Wu et al., 2016] and semantic parsing model with feedback SPF [Iyer et al., 2017]. Note that we do not compare against cell-based data querying approaches [Neelakantan et al., 2016; Yin et al., 2016], because they are only applicable to small tables while our testing databases contain way tens of thousands records.

Performance Metrics: We examine the quality of translation using three types of metrics. First, we report the token-level BLEU following [Yin and Neubig, 2017] to measure the quality of translation. Second, given the groundtruth SQL query $q$ and the predicted one $\widehat{q}$, we measure query accuracy as the fraction of queries with identical returned tuples. This is assessed by executing the predicted and groundtruth queries in the databases and examine their returned tuples. Third, we calculate the tuple recall and tuple precision of returned tuples of each $\widehat{q}$, by comparing these tuples against the outcomes from groundtruth $q$. The average precision and recall over all queries are reported. Note that the second metric focuses on query-level correctness, while the third metric evaluates individual tuples in query results. They are therefore numerically independent. All numbers reported in the experiments on $M A S$ and $I M D B$ are average of 5-fold cross validations.

Model Training and Optimization: In preprocessing, our approach uses NLTK to implement Conditional Random 
Proceedings of the Twenty-Seventh International Joint Conference on Artificial Intelligence (IJCAI-18)

\begin{tabular}{|l|c|c|c|}
\hline Hyperparameter & NMT & SPF & Ours \\
\hline \hline Batch Size & 128 & 100 & 128 \\
\hline Hidden Layer Size & 512 & 600 & 512 \\
\hline Encoder Layer & 2 & 2 & 2 \\
\hline Decoder Layer & 2 & 1 & 2 \\
\hline Optimizer & ADAM & ADAM & ADAM \\
\hline Learning Rate & 0.001 & 0.001 & 0.001 \\
\hline Bidirectional Encoder & Used & Used & Used \\
\hline Encoder Dropout Rate & 0.2 & 0.4 & 0.2 \\
\hline Decoder Dropout Rate & 0.2 & 0.5 & 0.2 \\
\hline Beam Search Size & - & 5 & - \\
\hline
\end{tabular}

Table 3: Hyerparameters of all approaches in experiments.

\begin{tabular}{|l|c|c|c|}
\hline Metric & NMT & SPF & Ours \\
\hline \hline BLEU & 83.2 & 38.1 & $\mathbf{8 5 . 2}$ \\
\hline Query Accuracy & 75.0 & 81.7 & $\mathbf{8 2 . 8}$ \\
\hline Tuple Recall & 77.4 & 83.7 & $\mathbf{8 4 . 1}$ \\
\hline Tuple Precision & 76.9 & 83.6 & $\mathbf{8 3 . 7}$ \\
\hline
\end{tabular}

Table 4: Results on Geo880 workload.

\begin{tabular}{|l|c|c|c|}
\hline Metric & NMT & SPF & Ours \\
\hline \hline BLEU & 82.6 & 82.8 & $\mathbf{8 3 . 0}$ \\
\hline Query Accuracy & 43.8 & 45.5 & $\mathbf{4 7 . 9}$ \\
\hline Tuple Recall & 62.7 & 64.6 & $\mathbf{6 6 . 2}$ \\
\hline Tuple Precision & 63.8 & 65.1 & $\mathbf{6 6 . 6}$ \\
\hline
\end{tabular}

Table 5: Results on MAS workload.

Fields [Lafferty et al., 2001] (CRFs) to annotate the natural language queries. The overall accuracy of annotation result is over $99.5 \%$. Therefore, the semantic features of the input words fed to the encoders are highly reliable. Our model is implemented in Tensorflow 1.2.0. The distributed representations of the words in the dictionary are automatically calculated and optimized by Tensorflow. We optimize the hyerparameters in all approaches and use the configuration with best results. The result hyperparameters are listed in Table 3 . Experimental Results: We report the experimental results on three databases in Tables 4, 5 and 6 resepctively. In terms of translation quality, our model achieves the highest BLEU on Geo880 and MAS while SPF performs the best on IMDB. The BLEU of SPF on Geo880 (38.1) is much lower than that of the other methods. This is because SPF uses templates to enlarge the training data significantly. Thus it tends to generate queries following those templates, which although returns the identical results but contains redundant components in the predicted query. In terms of quality of returned tuples by predicted queries, our model achieves the highest query accuracy on all three databases, i.e., the highest percentage of predicted queries with identical returned tuples. It is a significant improvement over the existing methods. The system could return completely right answers to over $80 \%$ of the questions on Geo880 and IMDB databases. Although the query accuracy of all approaches is below $50 \%$ on $M A S$ database, due to the high complexity on both schema and content, the recall and precision of the outcomes are all above $60 \%$. It implies that there remains certain utility even when the translation results contain errors. An interesting observation on the results over $I M D B$ database is: although SPF achieves the highest BLEU, the accuracy on query results by SPF and our approach are almost identical. It shows that translation quality, as used as

\begin{tabular}{|l|c|c|c|}
\hline Metric & NMT & SPF & Ours \\
\hline \hline BLEU & 85.7 & $\mathbf{8 6 . 7}$ & 85.7 \\
\hline Query Accuracy & 91.7 & 95.4 & $\mathbf{9 7 . 2}$ \\
\hline Tuple Recall & 96.9 & $\mathbf{9 7 . 8}$ & 97.5 \\
\hline Tuple Precision & 96.9 & $\mathbf{9 7 . 5}$ & $\mathbf{9 7 . 5}$ \\
\hline
\end{tabular}

Table 6: Results on $I M D B$ workload.

\begin{tabular}{|l|c|c|c|}
\hline Metric & - Short & - Long & - State \\
\hline \hline BLEU & 0.37 & -0.21 & 0.60 \\
Query Accuracy & -1.82 & -1.82 & -0.91 \\
Tuple Recall & -2.15 & -1.57 & -1.58 \\
Tuple Precision & -1.97 & -2.10 & -1.52 \\
\hline
\end{tabular}

Table 7: Evaluation of individual component on training set of Geo880 workload using 5-fold cross-validation. Difference between the "simpler" model and our original one are reported.

the golden standard in traditional machine translation tasks, may not be the best metric for our problem setting.

We conduct ablation studies on the training set of Geo880 (Table 7) and find that short/long-term dependencies (Short/Long) and grammar state (State) help improve quality of returned tuples in terms of query accuracy and tuple recall/precision. However, short-term dependencies and grammar state have negative effect on BLEU, i.e., the predicted queries are more similar to the groundtruth in the token level but are less accurate. This further implies that BLEU may not be the best metric for our problem setting.

\section{Conclusion}

In this paper, we present a new encoder-decode framework designed for translation from natural language to structured query language (SQL). The core idea is to deeply integrate the known grammar structure of SQL into the neural network structure used by the encoders and decoders. Our results show significant improvements over baseline approaches for standard machine translation, especially on the accuracy of outcomes by executing the SQL queries on real databases. It greatly improves the usefulness of natural language interface to relational databases.

Although our technique is designed for SQL outputs, the proposed techniques are generically applicable to other languages with BNF grammar structures. As future work, we will extend the usage to automatic programming, enabling machine learning systems to write programs, e.g., in C language, based on natural language inputs.

\section{Acknowledgements}

This research was supported in part by NSFC-Guangdong Joint Found (U1501254), Natural Science Foundation of China (61472089), Guangdong High-level Personnel of Special Support Program (2015TQ01X140), Pearl River S\&T Nova Program of Guangzhou (201610010101), Science and Technology Planning Project of Guangzhou (201604016075), Natural Science Foundation of Guangdong (2014A030306004, 2014A030308008), Science and Technology Planning Project of Guangdong (2015B010108006, 2015B010131015). And it was done when Zhang and Yang were with Advanced Digital Sciences Center, supported by 
the National Research Foundation, Prime Minister's Office, Singapore under its Campus for Research Excellence and Technological Enterprise (CREATE) programme.

\section{References}

[Castano et al., 1997] M Asunción Castano, Francisco Casacuberta, and Enrique Vidal. Machine translation using neural networks and finite-state models. Theoretical and Methodological Issues in Machine Translation, pages 160-167, 1997.

[Cho et al., 2014] Kyunghyun Cho, Bart Van Merriënboer, Caglar Gulcehre, Dzmitry Bahdanau, Fethi Bougares, Holger Schwenk, and Yoshua Bengio. Learning phrase representations using rnn encoder-decoder for statistical machine translation. arXiv preprint arXiv:1406.1078, 2014.

[Dong and Lapata, 2016] Li Dong and Mirella Lapata. Language to logical form with neural attention. In ACL, 2016.

[Gehring et al., 2017] Jonas Gehring, Michael Auli, David Grangier, Denis Yarats, and Yann N. Dauphin. Convolutional sequence to sequence learning. In $I C M L$, pages 1243-1252, 2017.

[Giordani and Moschitti, 2012] Alessandra Giordani and Alessandro Moschitti. Translating questions to SQL queries with generative parsers discriminatively reranked. In COLING, pages 401-410, 2012.

[Guu et al., 2017] Kelvin Guu, Panupong Pasupat, Evan Zheran Liu, and Percy Liang. From language to programs: Bridging reinforcement learning and maximum marginal likelihood. In $A C L, 2017$.

[Hochreiter and Schmidhuber, 1997] Sepp Hochreiter and Jürgen Schmidhuber. Long short-term memory. Neural computation, 9(8):1735-1780, 1997.

[Iyer et al., 2017] Srinivasan Iyer, Ioannis Konstas, Alvin Cheung, Jayant Krishnamurthy, and Luke Zettlemoyer. Learning a neural semantic parser from user feedback. In $A C L$, pages 963-973, 2017.

[Jia and Liang, 2016] Robin Jia and Percy Liang. Data recombination for neural semantic parsing. In $A C L, 2016$.

[Koncar and Guthrie, 1997] Nenad Koncar and Gregory Guthrie. A natural language translation neural network. In New Methods in Language Processing, pages 219-228, 1997.

[Lafferty et al., 2001] John Lafferty, Andrew McCallum, Fernando Pereira, et al. Conditional random fields: Probabilistic models for segmenting and labeling sequence data. In ICML, volume 1, pages 282-289, 2001.

[Li and Jagadish, 2014] Fei Li and HV Jagadish. Constructing an interactive natural language interface for relational databases. Proceedings of the VLDB Endowment, 8(1):7384, 2014.

[Liang et al., 2017] Chen Liang, Jonathan Berant, Quoc Le, Kenneth D. Forbus, and Ni Lao. Neural symbolic machines: Learning semantic parsers on freebase with weak supervision. In $A C L$, pages 23-33, 2017.
[Liang, 2016] Percy Liang. Learning executable semantic parsers for natural language understanding. Communications of ACM, 59(9):68-76, 2016.

[Long et al., 2016] Reginald Long, Panupong Pasupat, and Percy Liang. Simpler context-dependent logical forms via model projections. In $A C L, 2016$.

[Mikolov et al., 2013] Tomas Mikolov, Ilya Sutskever, Kai Chen, Greg S Corrado, and Jeff Dean. Distributed representations of words and phrases and their compositionality. In NIPS, pages 3111-3119, 2013.

[Mou et al., 2017] Lili Mou, Zhengdong Lu, Hang Li, and Zhi Jin. Coupling distributed and symbolic execution for natural language queries. In ICML, pages 2518-2526, 2017.

[Neelakantan et al., 2016] Arvind Neelakantan, Quoc V Le, Martin Abadi, Andrew McCallum, and Dario Amodei. Learning a natural language interface with neural programmer. arXiv preprint arXiv:1611.08945, 2016.

[Popescu et al., 2003] Ana-Maria Popescu, Oren Etzioni, and Henry A. Kautz. Towards a theory of natural language interfaces to databases. In IUI, pages 149-157, 2003.

[Rabinovich et al., 2017] Maxim Rabinovich, Mitchell Stern, and Dan Klein. Abstract syntax networks for code generation and semantic parsing. In $A C L$, pages 1139-1149, 2017.

[Roy et al., 2013] Senjuti Basu Roy, Martine De Cock, Vani Mandava, Swapna Savanna, Brian Dalessandro, Claudia Perlich, William Cukierski, and Ben Hamner. The microsoft academic search dataset and kdd cup 2013. In $K D D$ Cup 2013, page 1, 2013.

[Sutskever et al., 2014] Ilya Sutskever, Oriol Vinyals, and Quoc V Le. Sequence to sequence learning with neural networks. In NIPS, pages 3104-3112, 2014.

[Wu et al., 2016] Yonghui Wu, Mike Schuster, Zhifeng Chen, Quoc V Le, Mohammad Norouzi, Wolfgang Macherey, Maxim Krikun, Yuan Cao, Qin Gao, Klaus Macherey, et al. Google's neural machine translation system: Bridging the gap between human and machine translation. arXiv preprint arXiv: 1609.08144, 2016.

[Xiao et al., 2016] Chunyang Xiao, Marc Dymetman, and Claire Gardent. Sequence-based structured prediction for semantic parsing. ACL, 2016.

[Yin and Neubig, 2017] Pengcheng Yin and Graham Neubig. A syntactic neural model for general-purpose code generation. In $A C L$, pages 440-450, 2017.

[Yin et al., 2016] Pengcheng Yin, Zhengdong Lu, Hang Li, and Ben Kao. Neural enquirer: Learning to query tables in natural language. In IJCAI, pages 2308-2314, 2016.

[Zhou et al., 2016] Jie Zhou, Ying Cao, Xuguang Wang, Peng $\mathrm{Li}$, and Wei $\mathrm{Xu}$. Deep recurrent models with fastforward connections for neural machine translation. TACL, $4: 371-383,2016$. 